\title{
Liquid-Chromatographic Separation and Determination of Process-related Impurities, Including a Regio-Specific Isomer of Celecoxib on Reversed-Phase C18 Column Dynamically Coated with Hexamethyldisilazane
}

\author{
R. Nageswara RaO, ${ }^{* \dagger}$ S. Meena, ${ }^{* * *}$ D. NagaRaju, $*$ A. Raghuram RaO, ${ }^{* * *}$ and S. RaviKanth** \\ *HPLC/UV Group, Division of Analytical Chemistry, Discovery Laboratory, \\ Indian Institute of Chemical Technology, Hyderabad-500007, India \\ **Fluoro Organic Division, Indian Institute of Chemical Technology, Hyderabad-500007, India \\ ***Medicinal Chemistry Research Division, University College of Pharmaceutical Science, \\ Kakatiya University, Warangal-500009, India
}

\begin{abstract}
A simple and rapid reversed-phase high-performance liquid-chromatographic method for the separation and determination of process-related impurities of celecoxib (CXB) in bulk drugs and pharmaceuticals was developed. The separation of impurities viz., 4-methylacetophenone (I), 1-(4-methylphenyl)-4,4,4-trifluorobutane-1,3-dione (II), 4hydrazinobenzene sulfonamide (III) and a regio-specific isomer [3-(4-methylphenyl)-5-trifluoromethyl-1H-pyrazole-1-yl]benzenesulfonamide (IV), was accomplished on an Inertsil ODS-3 column dynamically coated with $0.1 \%$ hexamethyldisilazane (HMDS) in acetonitrile:water $(55: 45 \mathrm{v} / \mathrm{v})$ as a mobile phase and detection at $242 \mathrm{~nm}$ using PDA at ambient temperature. The chromatographic conditions were optimized by studying the effects of HMDS, an organic modifier, time of silanization and column temperature. The method was validated and found to be suitable not only for monitoring the synthetic reactions, but also to evaluate the quality of CXB.
\end{abstract}

(Received January 26, 2006; Accepted June 15, 2006)

\section{Introduction}

Selective COX-2 inhibitors are used to treat inflammatory conditions, such as rheumatoid arthiritis, osteoarthiritis, pain, cancer treatment and prevention of migraine. ${ }^{1,2}$ Celecoxib (CXB), 4-[5-(4-methylphenyl)-3-(trifluoromethyl)-1H-pyrazole1 -yl]benzenesulfonamide, is one of the COX-2 inhibitors primarily designed to minimize the adverse effects associated with classical nonspecific non-steroidal anti-inflammatory drugs (NSAIDs). ${ }^{3}$ In contrast to the known NSAIDs, CXB produces neither acute nor chronic gastrointestinal toxicities. Although it has proven bioavailability and established safety profile, the impact of the presence of impurities in bulk drugs and formulations remained unanswered. Some recent studies have thrown light on the fatal effects leading to their withdrawal from the market. ${ }^{4}$ Thus, there is a great potential for the development of new analytical methods, which enable us to identify and quantify all possible impurities of $\mathrm{CXB}$ further to evaluate their toxicological effects on human beings.

Spectrophotometric and chromatographic methods were routinely used for the analysis of $\mathrm{CXB}$ and its metabolites in biological fluids. ${ }^{5-7}$ Its purity was evaluated on a large number of commercial columns, including Novapak $\mathrm{C}_{18}$ with $0.01 \mathrm{M}$ potassium dihydrogen phosphate and acetonitrile $(45: 55 \mathrm{v} / \mathrm{v})$ as a mobile phase. ${ }^{8}$ However, these columns have shown very low capacity towards intermediates and severe peak tailing. These

$\dagger$ To whom correspondence should be addressed.

E-mail: rnrao@ins.iictnet.com; rnrao55@yahoo.com methods suffer not only from reproducibility, but also poor resolution between ortho and meta isomers of CXB. A Chiralpak-AD column was used to separate the positional isomers by LC. CXB and its impurities in pharmaceutical dosages were determined using a $25 \mathrm{mM}$ aqueous borate buffer as a background electrolyte and $25 \mathrm{mM}$ SDS as a surfactant by MEKC. ${ }^{9,10}$ LC-MS studies of CXB could detect four polar and one non polar regio-isomeric impurity in bulk drugs. ${ }^{11}$ Bebawy et al. used LC, TLC, and HPTLC to develop a stability indicating assay of $\mathrm{CXB}$ in the presence of degradation products. ${ }^{12,13}$ It could be seen from the literature that methods for separating the starting material as well as processing related impurities of CXB are not available. The present study is aimed at developing a chromatographic system capable of eluting and resolving CXB and its starting material, 4-methylacetophenone (I), process intermediates 1-(4-methylphenyl)-4,4,4-triflurobutane1,3-dione (II), 4-hydrazinobenzene sulfonamide (III), including the regio-specific isomer [3-(4-methylphenyl)-5-trifluoromethyl1H-pyrazole-1-yl]benzenesulfonamide (IV). The present paper describes a reversed-phase HPLC method using an Inertsil ODS-3 column dynamically coated with $0.1 \%$ HMDS in acetonitrile and water $(55: 45 \mathrm{v} / \mathrm{v})$ as a mobile phase and detection at $242 \mathrm{~nm}$ using PDA at ambient temperature.

\section{Experimental}

Materials and reagents

All reagents were of analytical-reagent grade unless stated otherwise. Glass-distilled and deionized water (Nanopure II, 
<smiles>CC(=O)c1ccc(C)cc1</smiles><smiles>[R]c1cc(C(F)(F)F)n([R])n1</smiles>

Regio-isomer IV

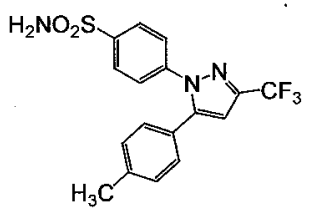

Celecoxib (CXB)

Fig. 1 Chemical structures of CXB and its related substances.

Barnsted, Iowa, USA), HPLC-grade acetonitrile (Ranbaxy, SAS Nagar, India) and, orthophosphoric acid (S.D. Fine Chem, Mumbai, India) were used. Samples of CXB, its reference standard (Unichem Laboratories, Ahmedabad, India) and impurities viz., 4-methylacetophenone (I) and ethyl trifluoro acetate (Fluka Chemika, GmbH, Buchs, Swizerland), 1-(4methylphenyl)-4,4,4-trifluorobutane-1,3-dione (II), 4-hydrazinobenzene sulfonamide (III), and a regio-isomer of celecoxib i.e., [3-(4-methylphenyl)-5-trifluoromethyle-1H-pyrazole-1-yl]benzenesulfonamide (IV), synthesized in our laboratory, were used.

\section{Apparatus}

The HPLC system was composed of two LC-10ATVP pumps, an SPD-M10AVP photodiode array detector, an SIL-10ADVP auto injector, a DGU-12A degasser and an SCL-10AVP system controller (all from Shimadzu, Kyoto, Japan). A reversed-phase Inertsil ODS-3 (GL Sciences Inc., Japan) column $(250 \times 4.6$ $\mathrm{mm}$ i.d., particle size $5 \mu \mathrm{m}$ ) was used for separation. The chromatographic and the integrated data were recorded using an HP-Vectra (Hewlett Packard, Waldbronn, Germany) computer system.

\section{Chromatographic conditions}

The Inertsil ODS-3 column was conditioned by equilibrating with $0.1 \%$ HMDS in acetonitrile and water $(55: 45 \mathrm{v} / \mathrm{v})$ as a mobile phase for $6 \mathrm{~h}$ before running the sample for analysis. Before delivering into the system it was filtered through 0.45 $\mu \mathrm{m}$ PTFE filter and degassed using a vacuum. The analysis was carried out under isocratic conditions at a flow rate of $1.0 \mathrm{~mL} / \mathrm{min}$ at room temperature $\left(28 \pm 1^{\circ} \mathrm{C}\right)$. Chromatograms were recorded at $242 \mathrm{~nm}$ using an SPD-M10A VP photo diode array detector.

\section{Analytical procedures}

Solutions of CXB $(1.0 \mathrm{mg} / \mathrm{mL})$ and impurities I, II, III and IV $(0.5 \mathrm{mg} / \mathrm{mL})$ were prepared in acetonitrile. Then, a $20-\mu \mathrm{L}$ volume of each solution was injected and chromatographed under the above conditions. The system suitability was evaluated by making five replicate injections of CXB (1.0 $\mathrm{mg} / \mathrm{mL}$ ) spiked with $0.1 \%$ of each impurity. The synthetic mixtures and process samples were analyzed under identical conditions. The quantities of impurities were calculated from their respective peak areas.

\section{Results and Discussion}

Figure 1 shows the chemical structures of CXB and its related

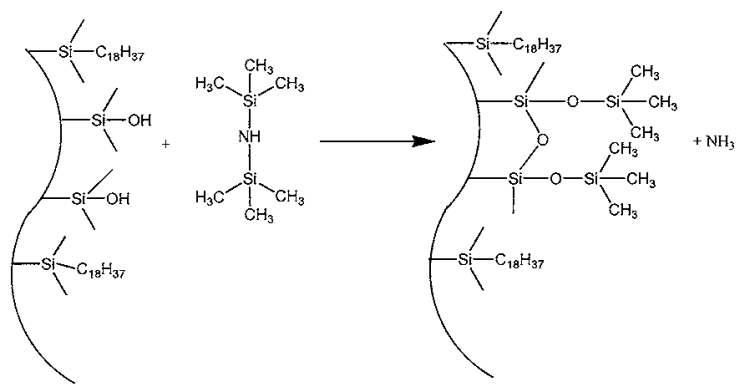

Fig. 2 Reaction of HMDS with free silanols.

substances (I, II, III and IV). Since the pharmacological and toxicological behavior of the regio-isomer (IV) is not known, its impact on COX-2 is yet to be evaluated. Structure-based docking studies were carried out on CXB and its 4 intermediates for their COX-2 inhibition in Silico. The program GOLD (Genetic Optimization for Ligand Docking), a genetic algorithm (version 2.2) from Cambridge Crystallographic Data Center, UK, was used to dock the inhibitors. The fitness of the compounds was found be in the order of CXB, IV, III, II and I, indicating that the binding of the regio-isomer is quite appreciable. If it exists as an impurity of $\mathrm{CXB}$, the chances of its binding with enzymes cannot be ignored, and a systemic study of the activity or the toxicity is required. Thus, it becomes quite important to develop analytical methods to separate and evaluate the pharmacological activity of $\mathrm{CXB}$ along with related substances, including its regio-isomer. As of today, to the best of our knowledge, there are no such methods available in the literature. We report for the first time on the separation and determination of impurities present in bulk drugs and formulations of CXB on a RP-column dynamically coated with HMDS.

\section{Method Development}

The impurities and $\mathrm{CXB}$ were subjected to separation on different $\mathrm{C}_{18}$ columns using buffers, such as acetate, TEA, and phosphate with acetonitrile as an organic modifier. However, compound II exhibited a characteristic tailing on all of the columns. This might not only be due to the high electropositive nature of $\mathrm{CF}_{3}$ but also to its ability to form strong hydrogen bonds with the residual silanol of the $\mathrm{C}_{18}$ materials. HMDS is a very active derivatizing reagent for silanization. It is used as an endcapping reagent to mask the sorption mechanism of the free silanols on the surface of the $\mathrm{C}_{18}$ silica. On the other hand, it seems to play a role as an ion-pair reagent in RP-LC separations. In order to study the mechanism of separating II, its analysis was carried out before and after silanization. It was observed that there was no change in the retention time (RT), but the peak tailing was reduced significantly. This shows that the HMDS could react with a free silanol group and not with the $C_{18}$ stationary phase. ${ }^{14,15}$ Further, it played a major role as an end capping reagent, not as an ion-pair. Finally, the peak tailing was overcome by converting the surface silanol into silanyl by dynamically coating the column with HMDS. The column was equilibrated for $6 \mathrm{~h}$ using $0.1 \%$ HMDS in acetonitrile. The peak tailing was greatly improved by coating the column with HMDS. The reaction of HMDS with free silanols is shown in Fig. 2.

Optimization of the chromatographic conditions

The HPLC conditions were optimized by studying the effects 


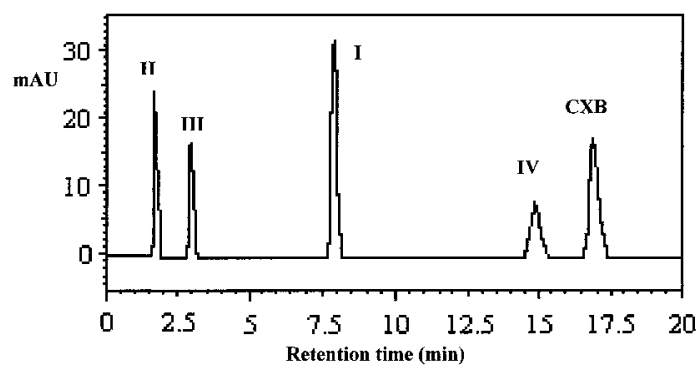

Fig. 3 HPLC chromatogram of a synthetic mixture containing CXB and its impurities (I, II, III and IV; $10 \mu \mathrm{g} / \mathrm{ml}$ each).

of the concentration of HMDS, organic modifier, buffer $\mathrm{pH}$, duration of prior silanization and temperature.

\section{Effect of concentration of HMDS}

The effect of HMDS was studied by varying its concentration in acetonitrile from $0.01 \%$ to $0.1 \%$. The results indicate that the $0.1 \%$ of HMDS optimum, and further increase in its concentration had no effect on the retention time of the test compounds. Thus, $0.1 \%$ HMDS was used for further optimization.

\section{Effect of an organic modifier}

The concentration of an organic modifier was varied from 45 to $55 \%$. Upon decreasing its concentration, CXB and IV were retained for more than $30 \mathrm{~min}$ in the column; $55 \%$ of the acetonitrile with $0.1 \%$ HMDS was found to be suitable with respect to lower retention and better separation.

\section{Effect of the buffer $p H$}

Buffers, such as glacial acetic acid, phosphate and triethanolamine, $\mathrm{pH}$ between 3.0 and 5.0, were tried to determine any improvement in the separation. The tailing of compound II was not only increased, but also merged with III, resulting in poor baseline separation. In addition, CXB was retained for a longer time on the column. Thus, water, without the addition of any buffer was selected as one of the main components of the mobile phase.

\section{Effect of the temperature}

The column was maintained at different temperatures in the range of $25-50^{\circ} \mathrm{C}$ in a thermostatic oven. As the temperature increased, compounds II/III and IV/CXB were merged and baseline separation was not achieved. The ambient conditions were found to be suitable for optimum separation.

\section{Effect of the equilibration time}

The duration required to attain dynamic equilibrium with a coating of HMDS was also studied. Prior silanizations for 3, 6 , and $12 \mathrm{~h}$ were carried out. Not much variation was observed, and it was concluded that $6 \mathrm{~h}$ of prior silanization was sufficient, because a further increase in the time caused a negligible variation of the retention time. The HMDS adhering to the column particles was removed by washing it with methanol for $2 \mathrm{~h}$.

A typical chromatogram of a synthetic mixture containing $\mathrm{CXB}$ and its impurities obtained under optimum conditions of separation is presented in Fig. 3. The peaks were identified by injecting and comparing with the retention times of the individual compounds. The elution order of the compounds was II, III, I, IV (regio-isomer) of CXB and CXB. The overall run
Table 1 System suitability data

\begin{tabular}{lcccr}
\hline Compound & $k^{\prime}$ & Tailing factor & RRT & \multicolumn{1}{c}{$R_{\mathrm{s}}$} \\
\hline I & 4.28 & 0.96 & 0.10 & 0.00 \\
II & 0.19 & 0.89 & 2.00 & 4.15 \\
III & 1.07 & 1.07 & 4.72 & 11.98 \\
IV & 9.06 & 0.83 & 0.91 & 27.96 \\
CXB & 10.01 & 1.09 & 1.0 & 2.38 \\
\hline
\end{tabular}

$\mathrm{RRT}$, relative retention time; $R_{\mathrm{s}}$, resolution; $k^{\prime}$, capacity factor.

time required for separation was within $20 \mathrm{~min}$. The method was substantiated with respect to the validation parameters, such as system suitability, precision, accuracy, linearity, limit of detection and quantification. The presence of impurities was tested in commercially available capsules of five different brands. The detected impurities were insignificant in quantities. The system suitability data are presented in Table 1.

\section{Validation}

\section{Precision}

The precision of the method was determined by injecting six solutions containing $0.1 \%$ of each impurity (i.e., I, II, III and IV) spiked to CXB and the RSD (\%) values of the retention time, peak area, tailing factor and the resolution were calculated for CXB and its impurities. The RSD (\%) was found to be between 0.15 and 1.2 .

\section{Accuracy}

The recoveries of compounds I, II, III and IV were assessed by spiking the CXB with each of the impurities at 6 different levels ranging from $0.5-1.5 \%$. The recovery range and RSD (\%) for all impurities were $89.94-106.94 \%$ and $0.29-1.7 \%$, respectively.

\section{Linearity}

The linearity of the peak area versus the concentration was studied in the range of 0.25 to $1.0 \mu \mathrm{g} / \mathrm{mL}$ for compounds I, II, III, IV and CXB. The data were subjected to statistical analysis using a linear-regression least-squares method. The calibration curves were found to be linear with a good correlation coefficient of 0.998 .

\section{Limits of detection and quantification}

The limits of detection (LOD) and quantification (LOQ) were determined by measuring the magnitude of the analytical background response by injecting blank samples; also the signal to noise ratio $(S / N)$ was calculated for each compound by injecting a series of diluted solutions until $S / N$ ratios of $2-3$ for LOD and 9.5 - 10.4 for LOQ were obtained.

\section{Stability}

The stability of CXB and its impurities in the mobile phase containing acetonitrile and water with $0.1 \%$ HMDS was evaluated by analyzing solutions spiked with impurities at $0.1 \%$ of the specification level. The solutions were tested after $48 \mathrm{~h}$ at room temperature; the results demonstrated that the samples were stable under these conditions. Simultaneously, the solution in the mobile phase was stored for $24 \mathrm{~h}$ in a refrigerator at $4^{\circ} \mathrm{C}$ and chromatographed the next day. No significant changes were observed in the chromatogram. 
Table 2 Robustness data

\begin{tabular}{|c|c|c|c|c|}
\hline \multirow{2}{*}{ Compound } & \multicolumn{4}{|c|}{ Retention time } \\
\hline & 15 days & 30 days & 45 days & 60 days \\
\hline \multirow[t]{3}{*}{ I } & 8.35 & 7.78 & 8.12 & 8.05 \\
\hline & 8.01 & 8.19 & 8.28 & 8.12 \\
\hline & 8.24 & 8.06 & 8.10 & 8.30 \\
\hline Mean $\pm S D$ & $8.11 \pm 0.17$ & $8.01 \pm 0.21$ & $8.11 \pm 0.10$ & $8.16 \pm 0.13$ \\
\hline \multirow[t]{3}{*}{ II } & 2.06 & 1.98 & 1.97 & 2.02 \\
\hline & 2.01 & 2.20 & 2.10 & 2.30 \\
\hline & 2.20 & 2.04 & 2.03 & 2.05 \\
\hline Mean \pm SD & $2.12 \pm 0.07$ & $2.28 \pm 0.11$ & $2.03 \pm 0.10$ & $2.12 \pm 0.15$ \\
\hline \multirow[t]{3}{*}{ III } & 2.71 & 2.98 & 2.67 & 2.91 \\
\hline & 2.76 & 2.88 & 2.71 & 2.85 \\
\hline & 2.94 & 2.78 & 2.92 & 2.70 \\
\hline Mean \pm SD & $2.80 \pm 0.12$ & $2.88 \pm 0.10$ & $2.77 \pm 0.13$ & $2.82 \pm 0.11$ \\
\hline \multirow[t]{3}{*}{ IV } & 15.08 & 15.04 & 15.12 & 15.14 \\
\hline & 15.15 & 15.24 & 15.26 & 15.05 \\
\hline & 14.90 & 15.11 & 15.06 & 15.36 \\
\hline Mean \pm SD & $15.10 \pm 0.13$ & $15.13 \pm 0.10$ & $15.15 \pm 0.10$ & $15.18 \pm 0.16$ \\
\hline \multirow[t]{3}{*}{$\mathrm{CXB}$} & 16.52 & 16.31 & 16.48 & 16.35 \\
\hline & 16.42 & 16.58 & 16.72 & 16.48 \\
\hline & 16.71 & 16.42 & 16.55 & 16.54 \\
\hline Mean $\pm S D$ & $16.55 \pm 0.15$ & $16.44 \pm 0.14$ & $16.58 \pm 0.12$ & $16.46 \pm 0.10$ \\
\hline
\end{tabular}

\section{Robustness}

The robustness of the method was evaluated to study whether the column was affected by silanization with HMDS, and also to know its long-term behavior towards the test compounds. A long-term study was carried out for 60 days and at 15 day intervals, CXB along with compounds I, II, III and IV were chromatographed, and the variation in RT was evaluated. The mean RT and the standard deviation (SD) are presented in Table 2. The results indicated that the method was robust in terms of stability of the column.

\section{Conclusion}

The separation of CXB and its process-related impurities on different $\mathrm{C}_{18}$ columns with different mobile phases was tried. The chromatographic conditions were optimized and the effects of the concentration of HMDS, organic modifier, time of prior silanization and temperature were studied. The developed method was simple and useful for monitoring the potential impurities of CXB. It provides a comprehensive procedure for determining the impurities that originated from the synthesis of $\mathrm{CXB}$ in a manufacturing unit. The method was found to be linear, accurate, reproducible and capable of separating impurities associated with the synthesis of CXB. The method can be used for process development as well as quality assurance of CXB in bulk drugs as well as formulations.

\section{Acknowledgements}

The authors wish to thank Dr. J. S. Yadav, Director, Dr. M. Vairamani, Head, Analytical Chemistry Division, Indian Institute of Chemical Technology, Hyderabad and G. Krishna Mohan, Principal, University College of Pharmaceutical Sciences, Kakatiya University, Warangal for encouragement and permission to communicate the results for publication. We thank Dr. B. Narsaiah, Fluoro Organics Division for valuable discussion and help rendered throughout the work and one of the authors (S. M.) wishes to thank AICTE, New Delhi for granting a fellowship under QIP.

\section{References}

1. D. Clemett and K. L. Goa, Drugs, 2000, 59, 957.

2. W. Cannon, Drugs of Today, 1999, 35, 487.

3. A. Graul, A. M. Martel, and J. Castner, Drugs of Future, 1997, 22, 711.

4. N. M. Davies and F. Jamali, J. Pharm. Sci., 2004, 7, 332.

5. R. N. Saha, C. Sajeev, P. R. Jadhav, S. P. Patil, and N. Srinivasan, J. Pharm. Biomed. Anal., 2002, 28, 741.

6. P. Damani, M. Bearzotti, and M. A. Cabezon, Anal. Bioanal. Chem., 2003, 376, 141.

7. M. M. Ghoneim and A. M. Beltagi, Talanta, 2003, 60, 911.

8. M. K. Srinivasu, Ch. L. Narayana, D. S. Rao, and G. O. Reddy, J. Pharm. Biomed. Anal., 2000, 22, 945.

9. D. S. Rao, M. K. Srinivasu, Ch. L. Narayana, and G. O. Reddy, J. Pharm. Biomed. Anal., 2001, 25, 21.

10. M. K. Srinivasu, D. S. Rao, and G. O. Reddy, J. Pharm. Biomed. Anal., 2002, 28, 493.

11. U. Satanarayana, D. S. Rao, Y. R. Kumar, J. M. Babu, P. R. Kumar, and J. T. Reddy. J. Pharm. Biomed. Anal., 2004, 35,951

12. L. I. Bebawy, A. A. Moustafa, and N. F. Abo-Talib, J. Pharm. Biomed. Anal., 2002, 27, 779.

13. P. M. Dhabu and K. G. Akamanchi, Drug Dev. Ind. Pharm., 2002, 28, 815.

14. F. Grossmann, V. Ehvald, C. D. Fresne von Hoohenesche, and K. K. Unger, J. Chromatogr., A, 2001, 910, 223.

15. J. J. Pesek, M. T. Matyska, and R. J. Yu, J. Chromatogr., A, 2002, 947, 195. 